\title{
Respiration and body movement analysis during sleep in bed using hetero-core fiber optic pressure sensors without constraint to human activity
}

\author{
Michiko Nishyama, Mitsuo Miyamoto, and Kazuhiro Watanabe \\ Soka University, Faculty of Engineering, Information Systems Science, 1-236 Tangi-machi, Hachioji, \\ Tokyo 192-8577, Japan
}

\begin{abstract}
We describe respiration monitoring in sleep using hetero-core fiber optic pressure sensors. The proposed hetero-core fiber optic sensor is highly sensitive to macrobending as a result of the core diameter difference due to stable single-mode transmission. Pressure sensors based on hetero-core fiber optics were fabricated to have a high sensitivity to small pressure changes resulting from minute body motions, such as respiration, during sleep and large pressure changes, such as those caused by a rollover. The sensors are installed in a conventional bed. The pressure characteristic performance of all the fabricated hetero-core fiber optic pressure sensors is found to show a monotonic response with weight changes. A respiration monitoring test in seven subjects efficiently demonstrates the effective use of eight hetero-core pressure sensors installed in a bed. Additionally, even in the case of different body postures, such as lying on one's side, a slight body movement due to respiration is detected by the hetero-core pressure sensors. @ 02011 Society of Photo-Optical Instrumentation Engineers (SPIE). [DOI: 10.1117/1.3528008]
\end{abstract}

Keywords: fiber optic sensor; respiration monitoring; sleep; unconstrained; weight.

Paper 10271R received May 21, 2010; revised manuscript received Oct. 24, 2010; accepted for publication Nov. 8, 2010; published online Jan. 12, 2011.

\section{Introduction}

Remote home monitoring services for biometrical information can provide caregivers remote access to the health condition of patients, so that they can be immediately informed of any abnormalities in their patients even at home. ${ }^{1,2}$ These technologies may give patients better control of their physical and mental health, as well as reduce care burden and visits to hospitals. To reduce the burden in caring for both home and hospital patients during long-term monitoring, biometric information, such as pulse, respiration rhythm, and heart beat, should be measured without constraint and without affecting the patients' daily activities. Therefore, many types of unconstrained biometric signal monitoring for home use have been proposed. . $^{3,4}$

With the current fast-paced lifestyle, it is difficult for people to get sufficient sleep. Sleep enables the brain to recover from fatigue and thus is required to maintain the health of both mind and body. Therefore, monitoring the sleep state without having to rouse the subjects and without constraining their daily activities would play an important role in health management.

Overnight polysomnography (PSG) inspection is a standard for the diagnosis of sleep apnea syndrome ${ }^{5}$ (SAS). PSG can record some biometric properties, such as airflow in the mouth and nose and movements of the chest and abdomen due to respiration, through a number of sensors attached to a patient's body. However, measurement systems including sensors and data analysis are less cost effective owing to the need for human resources and equipment. Thus, other approaches have been proposed, namely, oxyhemoglobin saturation detection us-

Address all correspondence to: Michiko Nishiyama, Faculty of Engineering, Soka University, Information Systems Science, 1-236 Tangi-machi, Hachioji, Tokyo 192-8577, Japan. Tel: 81-42-691-9427; Fax: 81-42-691-9427; E-mail: mnishiya@soka.ac.jp. ing pulse oximetry ${ }^{6}$ and fiber-grating camera-based detection. ${ }^{7}$ Fiber-grating camera-based sensor detection measures the upand-down motion at approximately 100 sampling points over the upper body; as a result, it can even detect respiratory movements.

In contrast to conventional health-monitoring embedding techniques, the use of pressure-sensitive sensors installed in a bed enables respiration monitoring during sleep, thereby enabling unconstrained daily activities for patients at home. Respiration monitoring employing the combined use of a pressure sensor and a standard bed has been conventionally proposed and developed as a multipoint polypiezofilm sensing, ${ }^{8}$ pneumaticsbased method that uses a thin, air-sealed cushion and a bed mattress. ${ }^{9}$ The pneumatic method requires air refills, since the air inside the mattress leaks during long-term use, and as a result, poses a maintenance difficulty. It was then proposed that this method be improved using a silicon rubber tube instead of an air mattress, because of the long-term usability of such a tube. $^{10}$

On the other hand, optical fiber sensors have several advantages for unconstrained respiration detection during sleep, such as flexibility, light weight, immunity to electromagnetic interference compared to electric sensors such as polypiezofilm sensors, and ease of installation in a bed. For conventional fiber optic sensors, a plastic optical fiber (POF) can detect bending action on an optical intensity basis and has been used in respiration monitoring in bed in combination with the sensors. ${ }^{11}$ However, POF generally has a multimode transmission line and is susceptible to the effects of the external disturbances such as bending and pressure.

$1083-3668 / 2011 / 16(1) / 017002 / 7 / \$ 25.00$ (C) 2011 SPIE 


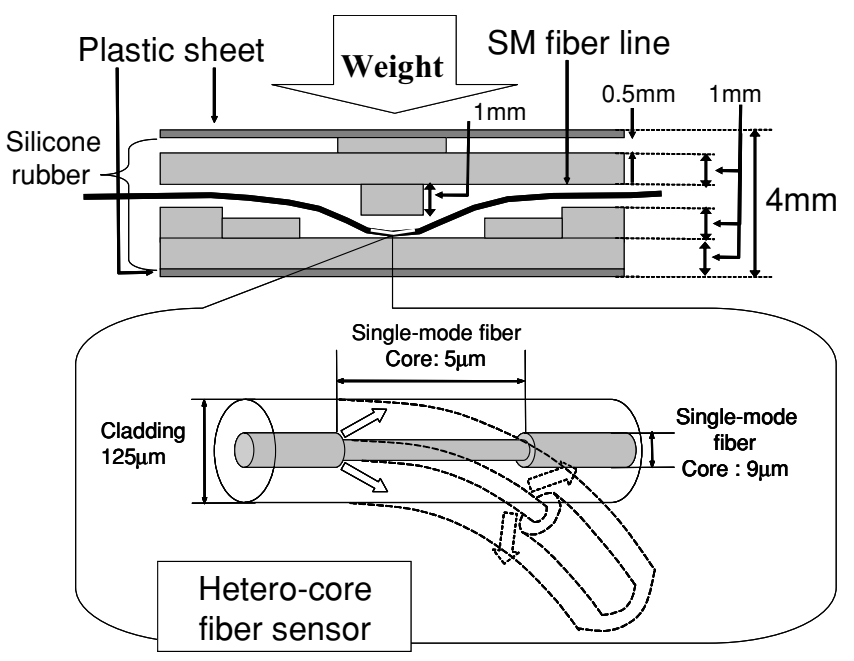

Fig. 1 Structure of a hetero-core fiber optic weight sensor made of silicone rubber and thin plastic sheets.

Thus far, we have proposed and developed hetero-core fiber optic bending sensors ${ }^{12-16}$ with stable single-mode transmission compared to POF sensors. The hetero-core fiber sensor is highly sensitive to moderate bending actions with optical loss changes and is independent of temperature fluctuation. Therefore, compared with the use of a conventional respiration-monitoring system, the hetero-core fiber technique is feasible for the realization of a high-cost-performance sleep measurement system. We also developed a wearable motion capture system using hetero-core fiber bending sensors that can detect the flexion of body joints, such as the elbows, shoulders, and knees. ${ }^{14}$

In this paper, sleep respiration monitoring employing heterocore fiber optic pressure-sensitive sensors is described. The sensors were fabricated to have sensitivity to small pressure changes arising from small body motions such as respiration during sleep as well as to large pressure changes such as those arising from a rollover. Eight hetero-core fiber optic pressure sensors were fabricated and evaluated of their pressure characteristic performance. Additionally, a respiration monitoring test is demonstrated using eight hetero-core pressure sensors installed in a conventional bed for seven subjects.

\section{Fiber Optic Pressure Sensor for Respiration Monitoring during Sleep}

\subsection{Hetero-Core Fiber Optic Sensor}

As shown in Fig. 1, the hetero-core fiber optic sensor structure consists of a single-mode (SM) transmission line 9- $\mu \mathrm{m}$ core diameter and an inserted sensor portion of a $5-\mu \mathrm{m}$ core diameter, which is as short as a few millimeters in fusion splice. A transmission beam is partially leaked into the cladding layer at the hetero-core sensor portion. Additionally, the leakage light changes with the bending action of hetero-core portion, so that the bending property can be measured due to the change in transmitted loss. The hetero-core insertion loss is found ${ }^{12}$ to be low at less than $1 \mathrm{~dB}$. The tandem-connected hetero-core sensing technique was recently proposed. Its use can decrease the number of transmission lines for multipoint sensing systems owing to the hetero-core sensor having a low insertion loss. ${ }^{14}$

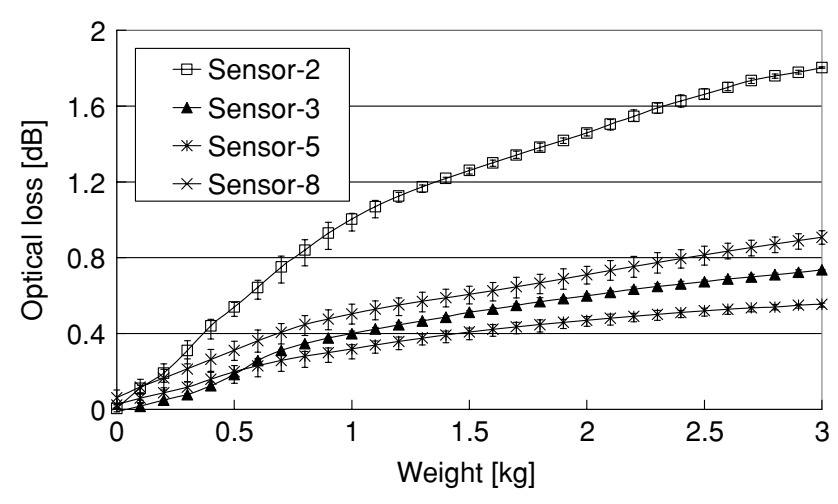

Fig. 2 Characteristics of hetero-core fiber optic weight sensors in the optical loss change with weights ranging from 0 to $3.0 \mathrm{~kg}$.

Additionally, we previously found that the hetero-core fiber sensor indicates monotonic and linear loss change characteristics with the bending action of hetero-core portion. ${ }^{12,13}$ In this paper, the hetero-core insertion length used is $2 \mathrm{~mm}$ because the heterocore fiber sensor has monotonic characteristics with relatively high sensitivity for bending.

\subsection{Characteristics of Weight Sensors Based on Hetero-Core Fiber Optics}

Figure 1 shows the hetero-core fiber pressure sensor structure made of silicone rubber, which is flexible and useful for longterm use. The hetero-core pressure sensor consists of a small segment of silicone rubber with a $1-\mathrm{mm}$ height and a $2-\mathrm{mm}$ width, which occupies the hetero-core portion, so as to induce the curvature change of the hetero-core portion by pressure on the upper and lower sides of the pressure sensor. In addition, four more silicone rubber segments of 3-mm width and 0.5- and 1-mm heights were placed on both sides of the silicone rubber sheets, which were $20-\times 20-\mathrm{mm}^{2}$ squares, for the pressure action to induce additional bending of the hetero-core portion, so that the sensitivity for pressure could be increased. Plastic sheets were attached on the silicone rubber sheets through their upper and bottom surfaces. The developed hetero-core pressure sensor measured $20 \times 20 \times 4 \mathrm{~mm}^{3}$, as shown in Fig. 1 .

The characteristics of the four hetero-core fiber pressure sensors $2,3,5$, and 8 , selected from eight hetero-core fiber optic pressure sensors to be fabricated for sleep respiration monitoring are indicated in Fig. 2, which shows average optical loss changes and standard deviations with weights ranging from 0 to $3.0 \mathrm{~kg}$, determined in five trials. As shown in Fig. 2, the four heterocore fiber pressure sensors exhibiting optical loss changes show monotonic characteristics with weight changes. The optical loss changes of the hetero-core pressure sensors $2,3,5$, and 8 for a weight change of $3.0 \mathrm{~kg}$ are $1.80,0.91,0.74$, and $0.55 \mathrm{~dB}$, respectively. Additionally, these pressure sensors appear capable of detecting relatively light weights of approximately $100 \mathrm{~g}$. Therefore, it is considered that minute pressure changes due to body movements during sleep respiration can be monitored using the proposed hetero-core pressure sensors. The weight of humans (often more than $50 \mathrm{~kg}$ ) is large and ranges over the pressure sensor's detectable range of 0 to $3.0 \mathrm{~kg}$. However, the detectable range is sufficient to measure respiration 
Nishyama, Miyamoto, and Watanabe: Respiration and body movement analysis during sleep in bed using hetero-core...

Table 1 Characteristics of hetero-core fiber optic weight sensors in the optical loss change with weight ranging from 0 to $3.0 \mathrm{~kg}$.

\begin{tabular}{lcccccccc}
\hline Sensor-No. & 1 & 2 & 3 & 4 & 5 & 6 & 7 & 8 \\
\hline Loss for full scale(dB) & 1.437 & 1.803 & 0.736 & 2.993 & 0.554 & 0.830 & 1.848 & 0.907 \\
Deviation (dB) & 0.073 & 0.085 & 0.021 & 0.426 & 0.061 & 0.107 & 0.082 & 0.070 \\
\hline
\end{tabular}

movement during sleep, because the weight distribution in the bed is expanded over the region of contact between the body, especially the head, backside, and hip, and the bed. Table 1 shows the average loss changes and deviations for five trials on the eight hetero-core fiber pressure sensors for a weight change in the range of 0 to $3.0 \mathrm{~kg}$. All the developed pressure sensors indicate monotonic optical loss changes with optical loss changes ranging from 0.74 to $2.99 \mathrm{~dB}$, as shown in Table 1 . However, they show deviations. This is because that the pressure sensors made of silicone rubber and glue had slightly different thickness and hardness. The differences are due to the differences in the amount of silicone glue used as well as in the pressure applied to the sensors by gradually adding a suspended weight, so that the weighted center of pressure appears to slightly change with each trial. The hetero-core fiber sensor itself has been indicated to have a high accuracy. ${ }^{12}$ Therefore, a slight weight change under constant pressure such as sleep respiration could be sufficiently detected by the hetero-core pressure sensors.

\section{Experimental Verification}

The experimental setup for sleep respiration monitoring is shown in Fig. 3. The light sources and detector employed were 1.3- $\mu \mathrm{m}$-wavelength LEDs and an optical power meter, which could measure eight channels simultaneously. The optical losses of the eight hetero-core pressure sensors were measured at a sampling frequency of $2 \mathrm{~Hz}$ through an analog-to-digital (A/D) converter with a PC. The eight hetero-core pressure sensors developed were fixed with Velcro tape to a bed measuring $180 \times 100 \mathrm{~cm}^{2}$, at locations corresponding to the chest and abdomen of a sleeping subject. During the monitoring, the sensors were covered with a thin cloth. Sleep respiration was continu-

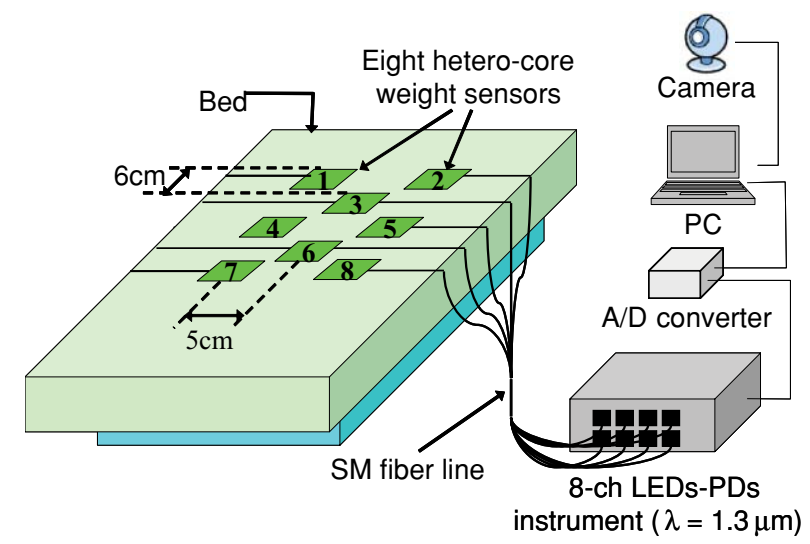

Fig. 3 Experimental setup for sleeping measurement based on eight hetero-core fiber optic weight sensors employing an eight-channel fiber power meter using LEDs and photodetectors (PDs). ously detected even when the subject rolls over, since the detector remains functional even with small distances between the pressure sensors and the subjects of 6 and $5 \mathrm{~cm}$ in the $x$ and $y$ directions, respectively, as shown in Fig. 3. Therefore, many sensing points can easily detect the respiration during sleep with the human moving in bed. Referential monitoring during sleep was achieved by video recording. In a series of respirationmonitoring tests during sleep, seven subjects in their 20s were tested. In the test, only the position of the pillow was specified relative to the position of sensors; on the other hand, there was no limitation on the body posture of the subjects. Figure 4 shows photographs of the developed pressure sensors and the eight hetero-core fiber optic pressure sensors placed on the bed for the sleep respiration test.

\section{Results and Discussion}

\subsection{Respiration Monitoring Test during Sleep}

Figure 5 shows real-time responses of the hetero-core fiber pressure sensors in the optical loss change during sleep for respiration with rollover and apnea phenomena. The experimental sleep tests were actually demonstrated during more than $6 \mathrm{~h}$ at night, and partially sampled for $30 \mathrm{~s}$ on the whole sleep respiration waveforms. In the respiration test during sleep, optical loss was initially set at $0 \mathrm{~dB}$ for the pressure sensor that was not to be weighted, and therefore could be induced by a slight weight change. As shown in Fig. 5, the hetero-core pressure sensors indicated a waveform of optical loss changes in a short time period of a few seconds. These were detected during respiration in sleep, which also induced constant optical losses of approximately 1.3 and $0.8 \mathrm{~dB}$ for sensors 6 and 4, respectively. This is because the weight that a body exerts on the sensors induces

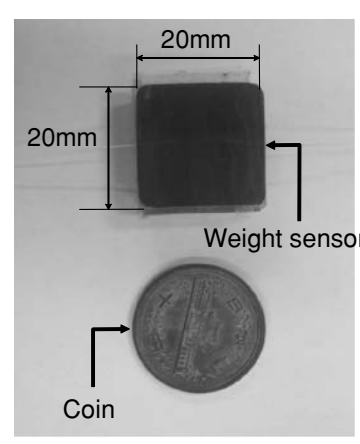

(a)

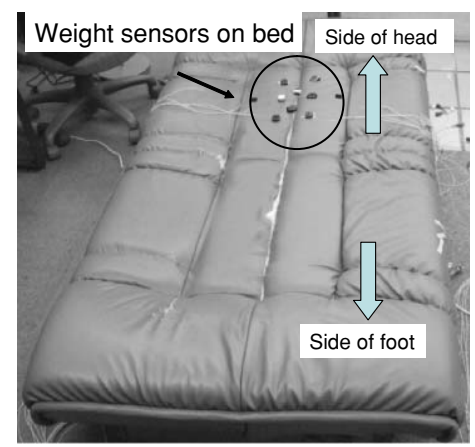

(b)
Fig. 4 Photos of proposed weight sensors for sleep respiration measurement: (a) the developed weight sensor and (b) eight hetero-core fiber optic weight sensors put on the bed to be used for the respiration test during sleep. 
Nishyama, Miyamoto, and Watanabe: Respiration and body movement analysis during sleep in bed using hetero-core...

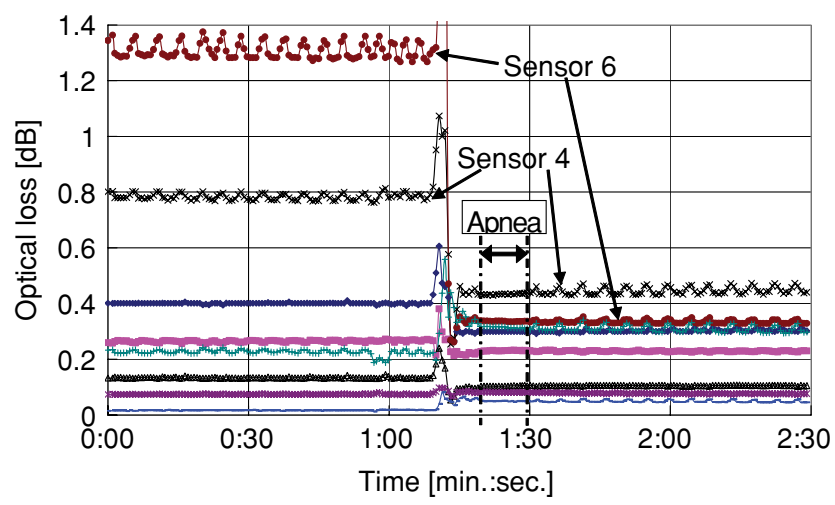

Fig. 5 Real-time responses of hetero-core fiber weight sensors in the optical loss change during sleep for respiration with rolling over and apnea phenomena.

constant optical losses. On the other hand, the sleep-monitoring result shows that the waveform was disturbed after periodical optical loss changes, which was due to a rollover as checked in the video recording. After a rollover, we found that the pressure sensors again detected respiration action, which differed from the respiration movement detected by other sensors before a rollover. Therefore, the proposed system could respond to the positional movement of a body due to a rollover, because the pressure sensors are widely distributed, as shown in Fig. 3. On the other hand, immediately after a rollover, no periodical loss changes are observed under a constant optical loss, and after a few tens of seconds, a waveform is again detected, as shown in Fig. 5. This result indicates that the system can recognize apnea and infrequent respiration during sleep and thus may be used in analyzing SAS.

Figure 6 shows the real-time responses of the eight heterocore fiber pressure sensors in terms of optical loss changes during sleep with reference to the average optical loss of the pressure sensors, which means that the initial loss of $0 \mathrm{~dB}$ should be set as the average optical loss in these tests. All of the heterocore pressure sensors detected body movements from respiration during the sleep of the subjects, indicated as optical loss periodical changes, as shown in Fig. 6. In particular, sensor 7 indicated that the amplitude of respiration waveform in terms of optical loss change is sufficient at approximately $0.09 \mathrm{~dB}$, as shown in Fig. 6. Additionally, the short-period periodical optical loss changes induced at constant optical losses are stable; therefore, the hetero-core fiber optic pressure sensor can stably measure body weight. On the other hand, the weight applied to the hetero-core pressure sensors during sleep was less than several kilograms, because the constant optical losses were near the range of the full-scale losses of the eight hetero-core pressure sensors, as shown in Table 1. Focusing on the results of sensors 1 and 7 in Fig. 6, the loss waveforms were out of phase for the time elapsed. This is because that the weight differences were induced among the hetero-core pressure sensors 1 and 7 installed at the thorax and in the abdominal lumbar region in bed, respectively. However, these sensors indicated the same time period of respiration; therefore, respiration monitoring in sleep could be attained. Additionally, the respiration test during sleep was successfully performed in seven healthy males, which means that a broadly distributed arrangement of the hetero-core
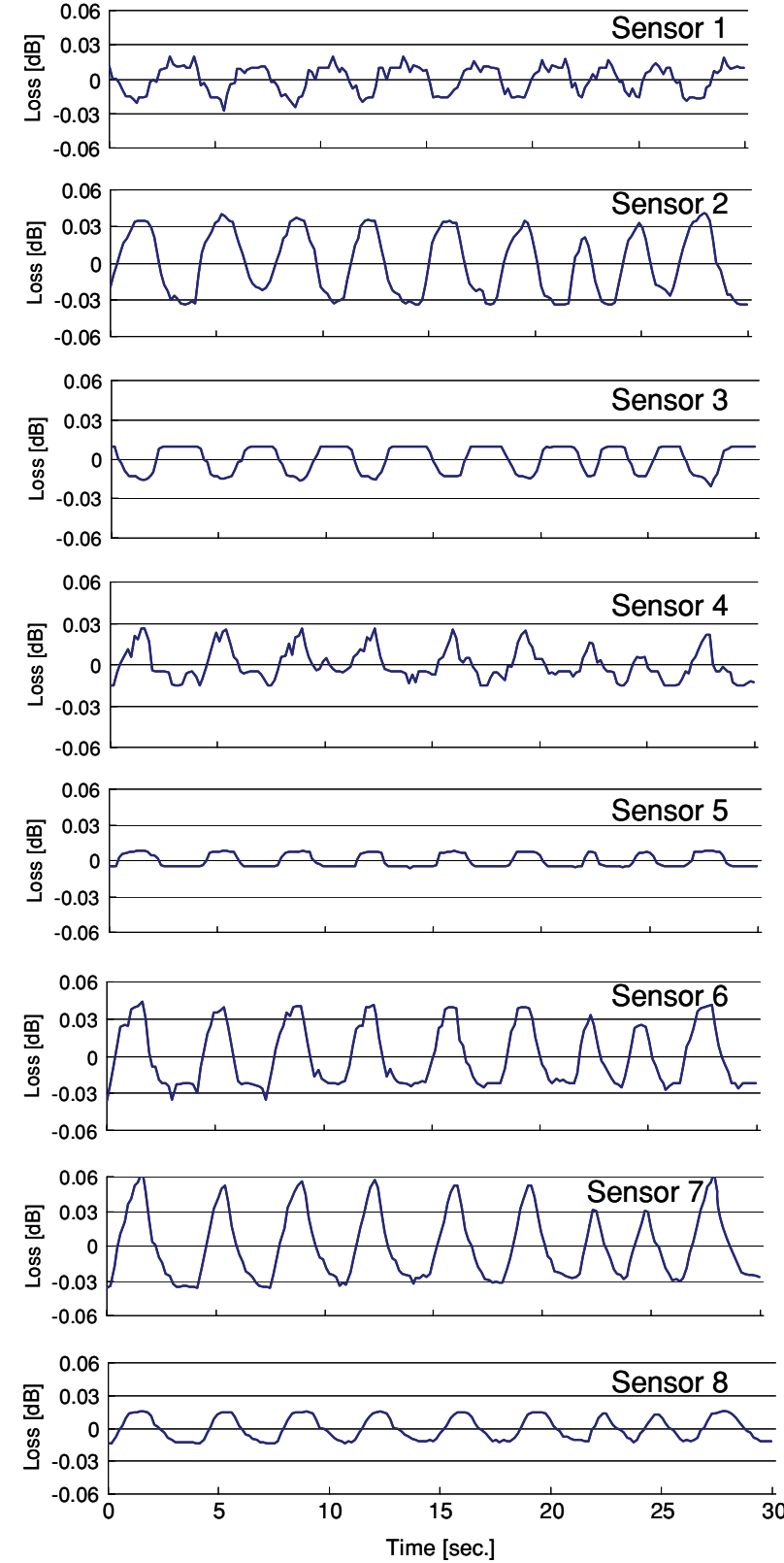

Fig. 6 Real-time responses of hetero-core fiber weight sensors in the optical loss change during sleep for subject A taking breaths repeatedly.

pressure sensors is useful for monitoring the various daily activities of humans during sleep. The other subject test indicated a maximum loss amplitude of approximately $0.1 \mathrm{~dB}$.

\subsection{Characteristics of Respiration Responses for Arrangement of Different Pressure Sensors}

To achieve unawareness and constraint-free respiration monitoring during sleep, it is important that the subject can use the respiration monitoring system with various body postures. To evaluate the characteristics of respiration responses of the hetero-core pressure sensors in the bed arranged at various positions, such as near the thorax or abdominal and lumbar regions, we tested respiratory monitoring using eight hetero-core 


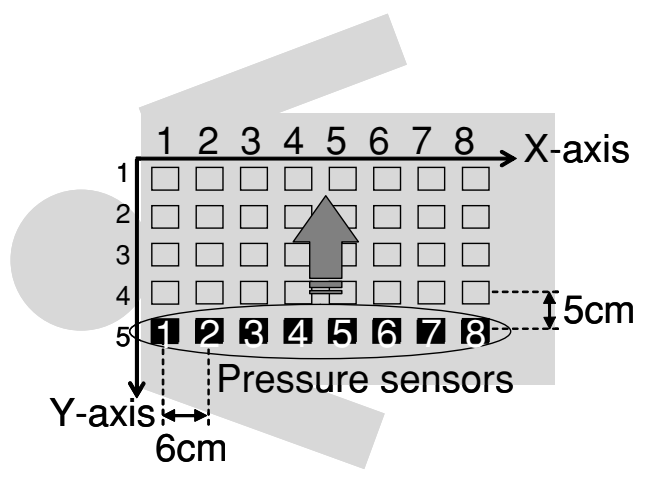

Fig. 7 Arrangement of weight sensors for the body position referring to sensor number for the respiration amplitude distribution test.

pressure sensors fixed on a flat floor for subjects A and B, as indicated in Fig. 7. As shown in the figure, sensors 5 and 8 were placed on the $x$ axis, parallel to the shoulder, and on the $y$ axis, respectively. The sensors were separately arranged at $5-\mathrm{cm}$ intervals in the $x$ direction and at 6-cm intervals in the $y$ direction. The pressure sensors closest to the head were located $6 \mathrm{~cm}$ from the shoulder. The position matrix of the sensors was defined as shown in Fig. 7, for example, sensor 1 in Fig. 7 was defined as $(X, Y)=(1,5)$.

Figure 8 shows the real-time response of the proposed heterocore pressure sensors in terms of optical loss at the position $(X, Y)=(7,3)$, which means that the sensors are near the thorax, on the back of the subject. Amplitude was defined in the respiration waveform shown in Fig. 8. The output waveform from the respiration test exhibited detection ability even for minute body motion; however, it showed fluctuation at relatively high frequencies owing to the noise floor from the detector electronics. The experimental result of the distributed weight pressure obtained using hetero-core pressure sensors, whose locations are shown in Fig. 7, is shown in Fig. 9 for subjects A and B. The loss amplitude of the sensors in sleep measurement is defined as the average amplitude of a waveform, as shown in Fig. 8. The sensor positions with the highest loss amplitudes were $(X, Y)$ $=(4,2)$ and $(3,5)$ and their loss amplitudes were 0.467 and $0.129 \mathrm{~dB}$ for subjects A and B, respectively. The number of pressure sensor positions necessary to detect respiration in subjects

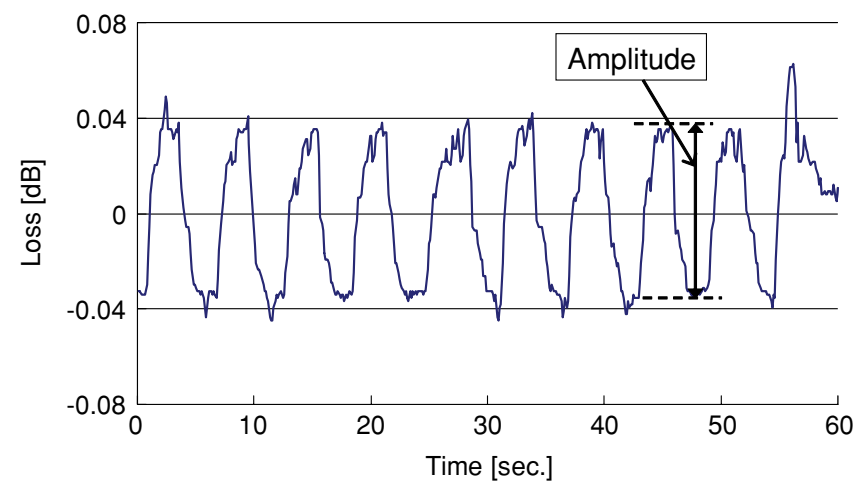

Fig. 8 Real-time response of the proposed hetero-core fiber weight sensor at the position $X=7$ and $Y=3$ (shown in Fig. 7) in the optical loss for respiration while lying on the back.

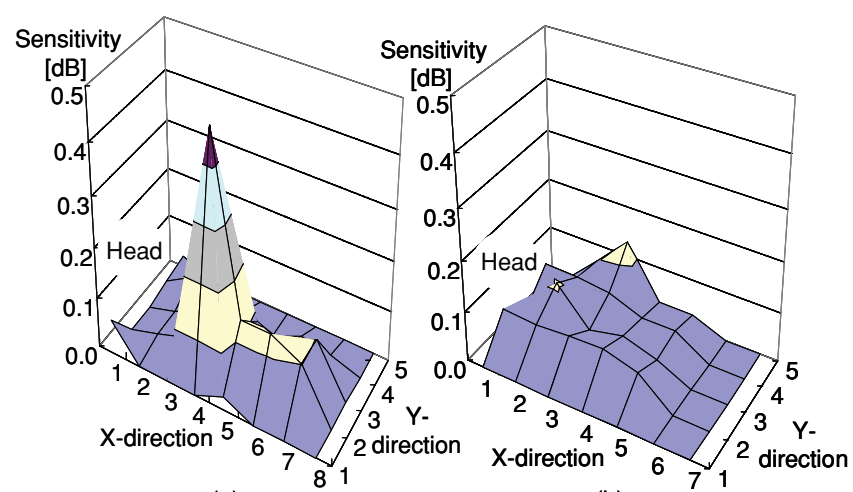

(a)

(b)

Fig. 9 Sensitivity distribution along the body of the weight sensors for respiration while lying on the back for subjects (a) A and (b) B.

A and B were 21 and 19, respectively. The area for respiration motion detection was relatively wide, therefore, by using several pressure sensors separately placed in a bed, the proposed monitoring system can be considered capable of detecting respiration motion even during body movements such as a rollover in various subjects.

\subsection{Characteristic Respiration Responses for Different Body Postures}

As shown in Fig. 10, we evaluated the effect of subject posture with reference to a subject's back, side, and stomach using eight hetero-core pressure sensors, whose positions were chosen so as to carry out high-sensitivity measurement based on previous experimental results, as shown in Fig. 9. Figures 11(a) to 11(c) show real-time responses of the eight hetero-core pressure sensors for respiration while lying on the back, side, and stomach, respectively. For all three postures, body movement with respiration was detected, as can be seen in Fig. 11. In Fig. 11(a), which shows data for lying on the back, sensors 1 and 2 detected respiration at sensitivities of approximately 0.1 and $0.05 \mathrm{~dB}$, respectively; however, sensor 8 had difficulty detecting respiration, because of its different weight position. On the other hand, in the case of the subject posture on the side, sensors 2 and 8 had sensitivities of approximately 0.1 and $0.2 \mathrm{~dB}$, respectively, for respiration. In contrast, sensor 1 was

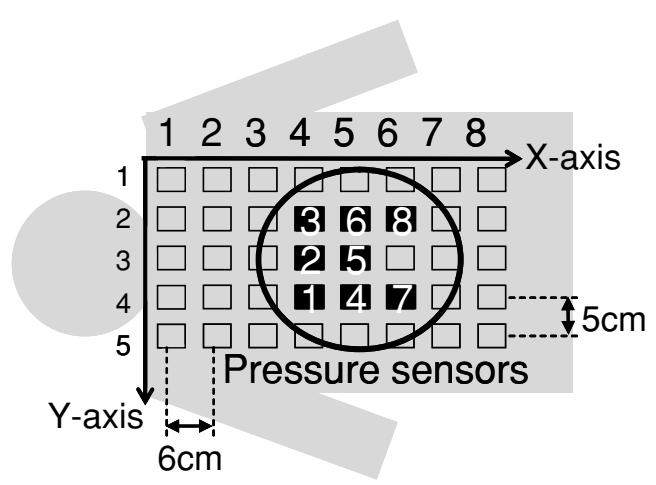

Fig. 10 Arrangement of weight sensors for the body position referring to sensor number for comparison of body posture, such as lying on the back, side, and stomach. 
Nishyama, Miyamoto, and Watanabe: Respiration and body movement analysis during sleep in bed using hetero-core...

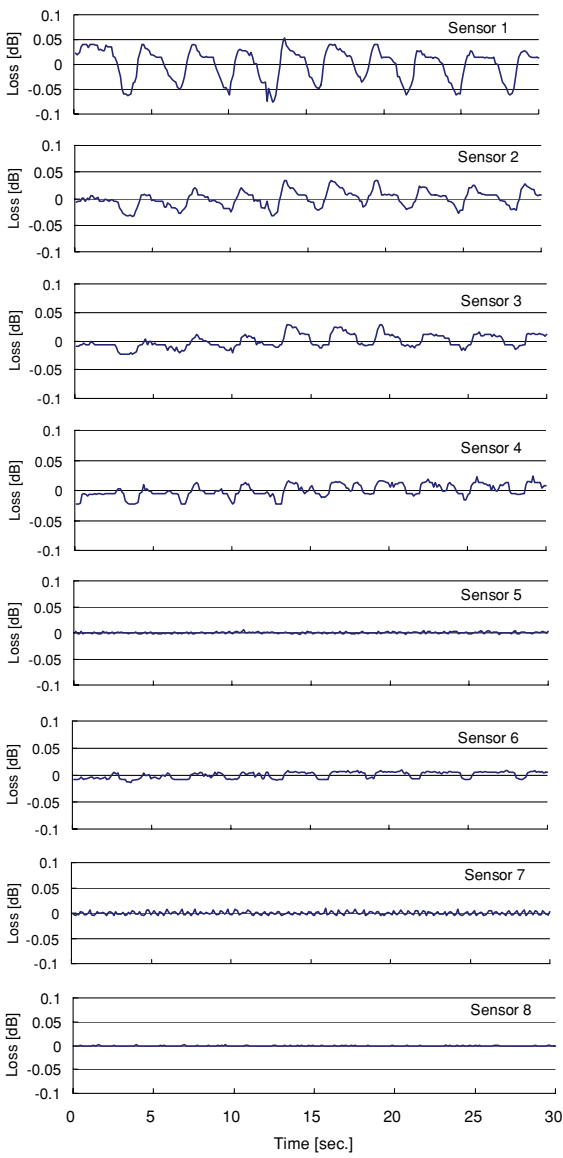

(a)
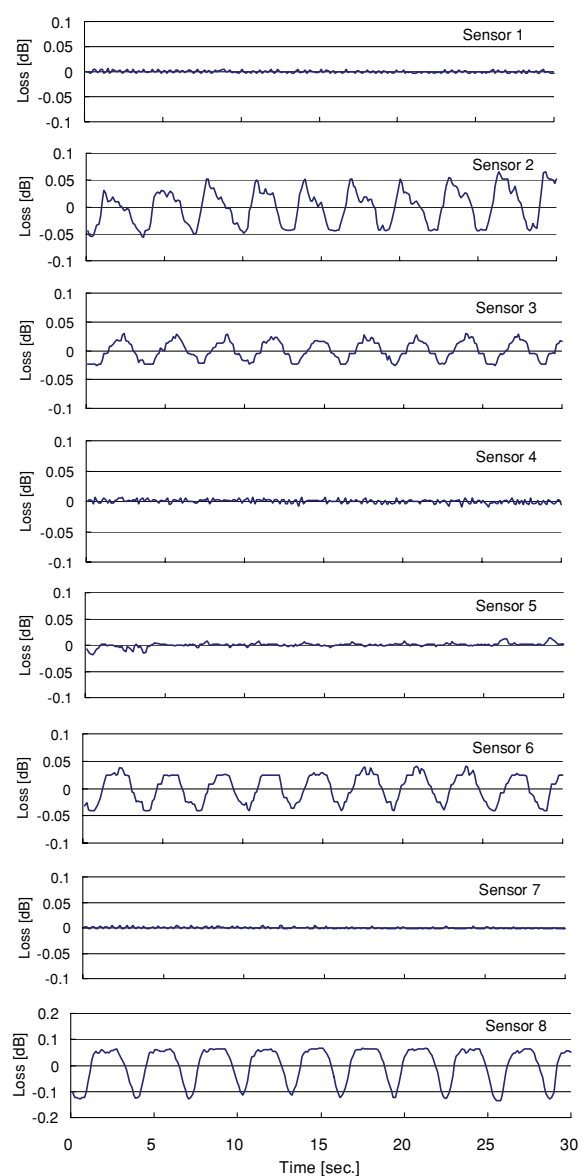

(b)
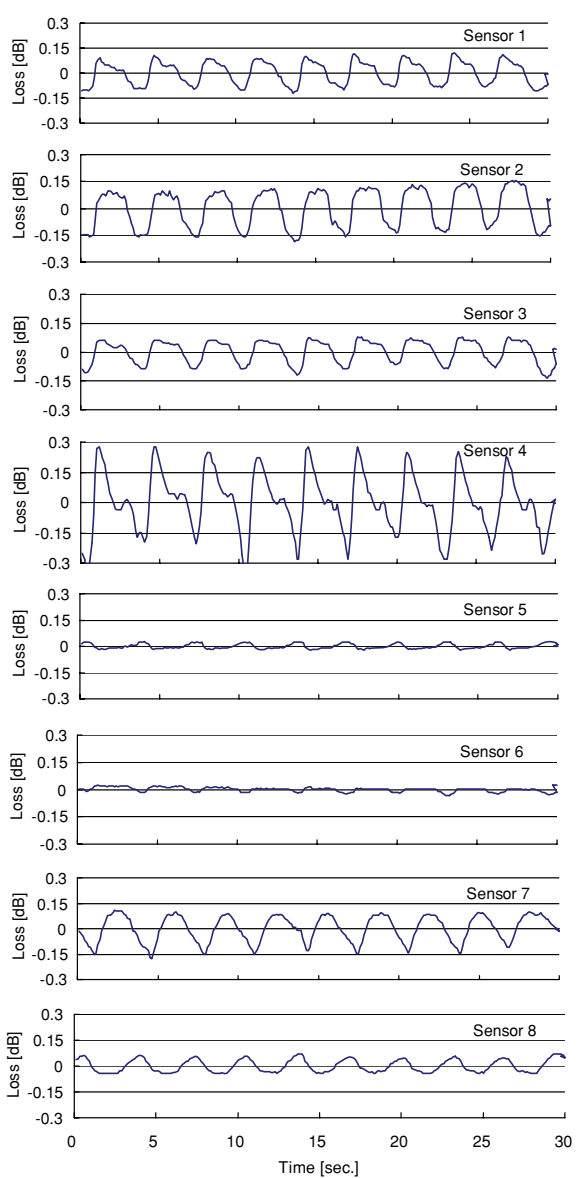

(c)

Fig. 11 Real-time responses of eight hetero-core fiber weight sensors in the optical loss change during respiration on the bed shown in Fig. 6(b) for subjects lying (a) on the back, (b) on the side, and (c) on the stomach.

insensitive to respiration motion, as indicated in Fig. 11(b). For the third posture on the stomach, all the sensors detected respiration, as shown in Fig. 11(c). These results show that sensor 8 was out-of-phase with sensors 1 and 2 for the time elapsed in the measured respiration waveform, for the test on the side. This is because sensor 8 is separated from sensors 1 and 2, which are close to each other. Additionally, it is considered that sensor 8 and sensors 1 and 2 are located near the stomach and chest, respectively, which means that the timings of expansion and contraction of the stomach and thorax are different. These results indicate that the hetero-core pressure sensors can detect minute weight changes connected to respiration, especially in the posture on the side; thus, they can be applied at various positions during sleep. Therefore, it is evident that a heterocore respiration-monitoring system during sleep can be useful in measuring daily activities without constraining the subjects.

\section{Conclusion}

We researched and developed hetero-core fiber optic pressure sensors for detecting minute body movements due to respiration during sleep. The hetero-core sensors were found to be highly sensitive and to obtain reproducible results for macrobending. The hetero-core weight sensor had a high sensitivity, that is, its optical loss changes ranged from 0.74 to $2.99 \mathrm{~dB}$ for a weight of $3.0 \mathrm{~kg}$.

The actual respiration monitoring test during sleep was successfully demonstrated for seven healthy male subjects using the eight hetero-core fiber optic pressure sensors developed. In addition, the hetero-core pressure sensors exhibited effective respiration monitoring in three postures, that is, on the back, side, and stomach, which means that the proposed sensors can respond to various body postures such as those associated with a rollover. Finally, an unconstrained and automatic respiration monitoring system based on hetero-core fiber sensing may provide help with health care in daily life, improve sleep conditions, and be used in analyzing SAS.

\section{Acknowledgments}

This research was partially supported by the Collaboration with Local Communities Project for Private Universities on Development of Ubiquitous-Monitoring-Network Based on Distributed Sensor Nodes using Local Positioning/Optical Sensory Nerves and their Industrial Applications: matching fund subsidy, 2006 to 2010, and by KAKENHI (Grant No. 22700482) of Grant-inAid for Young Scientists (B), 2010 to 2012, from MEXT (The Ministry of Education, Culture, Sports, Science and Technology of Japan). 


\section{References}

1. C. Lin, S. Young, and T. Kuo, "A remote data access architecture for home-monitoring health-care applications," Med. Eng. Phys. 29, 199204 (2007).

2. J. M. Kang, T. Yoo, and H. C. Kim, "A wrist-worn integrated health monitoring instrument with a tele-reporting device for telemedicine and telecare," IEEE Trans. Instrum. Meas. 55(5), 1655-1661 (2006).

3. T. Tamura, T. Togawa, M. Ogawa, and M. Yoda, "Fully automated health monitoring system in the home," Med. Eng. Phys. 20, 573-579 (1998).

4. S. Tanaka, Y. Matsumoto, and K. Wakimoto, "Unconstrained and noninvasive measurement of heart-beat and respiration periods using a phonocardiographic sensor," Med. Biol. Eng. Comput. 40, 246-252 (2002).

5. B. V. Vaughn and P. Giallanza, "Technical review of polysomnography," Chest 134, 1310-1319 (2008).

6. N. Nezer, A. H. Eliasson, C. Netzer, and D. A. Kristo, "Overnight pulse oximetry for sleep disordered breathing in adults," Chest $\mathbf{1 2 0}$, 625-633 (2001).

7. Y. Takemura, J. Sato, and M. Nakajima, "A respiratory movement monitoring system using fiber-grating vision sensor for diagnosing sleep apnea syndrome," Opt. Rev. 12(1), 46-53 (2005).

8. K. Niizaki, I. Nishidate, K. Uchida, and M. Kuwahara, "Unconstrained cardiorespiratory and body movement monitoring system for home care," Med. Bio. Eng. Comput. 43, 716-724 (2005).

9. K. Watanabe, T. Watanabe, H. Watanabe, H. Ando, T. Ishikawa, and K. Kobayashi, "Noninvasive measurement of heartbeat, respiration, snoring and body movements of a subject in bed via a pneumatic method," IEEE Trans. Biomed. Eng. 52(12), 2100-2107 (2005).

10. K. Watanabe, Y. Kurihara, and H. Tanaka, "Ubiquitous health monitoring at home-sensing of human biosignals on flooring, on tatami mat, in the bathtub, and in the laboratory," IEEE Sens. J. 9(12), 1847-1855 (2009).

11. S. Mitachi, D. Shiroishi, and M. Nakagawa, "Development of a sleep apnea syndrome sensor using optical fibers," in Proc. IEEE 20th LEOS Апnи. Meet., Vol. 1, pp. 294-295 (2007).

12. H. Sasaki, Y. Kubota, and K. Watanabe, "Sensitivity property of a hetero-core splice fiber optic displacement sensor," Proc. SPIE 5579, $136-143$ (2004).

13. M. Nishiyama, H. Sasaki, and K. Watanabe, "A deformation sensitive pad-structure embedded with hetero-core optic fiber sensors," Sens. Actuat. A 136(1), 205-211 (2007).

14. M. Nishiyama, H. Sasaki, and K. Watanabe, "Performance characteristics of wearable embedded hetero-core fiber sensors for unconstrained motion analyses," Trans. Soc. Instrum. Contr. Eng. 43(12), 1075-1081 (2007).

15. M. Nishiyama, H. Sasaki, and K. Watanabe, "Optical intensity-based measurement of multipoint hetero-core fiber sensors by the method of time-differentiation in optical loss," IEEE Sens. J. 8(7), 1055-1060 (2008).

16. M. Nishiyama and K. Watanabe, "Wearable sensing glove with embedded hetero-core fiber-optic nerves for unconstrained hand motion capture," IEEE Trans. Instrum. Meas. 58(12), 3995-4000 (2009). 